\title{
RESECTION WITH RESTORATION OF CONTINUITY IN THE TREATMENT OF CARCINOMA OF THE RECTUM AND RECTOSIGMOID
}

\author{
J. C. GOLIGHER, Ch.M., F.R.C.S. \\ Assistant Surgeon St. Mary's Hospital and St. Mark's Hospital for Diseases of the Rectum and Colon
}

The results of excision of the rectum for carcinoma by the modern abdomino-perineal operation are among the most gratifying in cancer surgery. In experienced hands the immediate operative mortality is certainly no greater than Io per cent. even when the range of operability has been broadened to include fully 80 per cent. or more of all the cases presenting for treatment, and of the operation survivors approximately one-half are alive and well five years later. Yet the excellence of these achievements is to some extent marred by the fact that all these patients are left with a permanent colostomy. True, the average patient soon learns to adapt himself to this disability and seldom suffers serious inconvenience or economic hardship on account of it, but it is an exceptional patient who would not give a good deal to be rid of this unnatural and objectional state of affairs. Under the circumstances, though the surgeon might wish to avoid a colostomy in these cases, it would certainly not be justifiable to accept a lowering of the prospect of complete eradication of the disease in order to do so, and it would be most deplorable if preservation of natural continence were to be allowed to deflect the surgeon from his main objective of curing the patient.

The problem, therefore, is whether retention of the anal sphincter apparatus can ever be practised without prejudicing the chances of radical removal of a rectal carcinoma. Obviously in many cases this is absolutely impossible. Where the growth actually invades the sphincters or lies close to them complete excision without sacrifice of the sphincter apparatus is quite out of the question. Likewise if the growth is situated in the lower one-third or half of the rectum where it is supported laterally and posteriorly by the levator muscles so that direct spread into these structures may readily occur, it is undoubtedly preferable that these muscles should be excised together with the rectum by a formal combined abdomino-perineal excision rather than conserved as in a sphincter-saving resection. But even if all the carcinomata arising in the extraperitoneal part of the rectum (i.e. below the peritoneal reflection) are regarded as unsuitable for sphincter preservation, this still leaves for consideration the intraO peritoneal growths, and, if to these are adde $\phi$ carcinomata of the rectosigmoid, the number cone cerned is quite large. Thus in a recent analysis of the site of the growth in a consecutive series of I,500 combined excision specimens at St. Mark's Hospital the writer found that in 713 , or 47.5 per cent., it lay entirely above the peritoneal reflection in the intraperitoneal part of the rectum or in the rectosigmoid (see Table I). Is it really necessary to sacrifice the sphincter apparatus in dealing radically with these growths ?

Some surgeons such as Rankin (1947), Allen (1946) and Gabriel (1948) reply most emphatically that it is, and, as their authority for this statement, quote the classical researches of Miles (1910). It may be recalled that Miles (19ro), on the basis of a most careful examination of operation specimens of rectal carcinoma, of recurrences after limited operations for this disease and of patients dying in the late stages of untreated rectal cancer, came to the conclusion that with a carcinoma anywhere in the rectum, even when quite early, lymphatic spread could take place in several different directions. These included spread downwards to the pararectal glands on the back of the rectum below the growth, lateralwards through glands on the levator muscles eventually to the internal iliac glands on the side wall of the pelvis, and upwards, not only along the main inferior haemorrhoidal and inferior mesenteric vessels but also to the paracolic glands lying in the

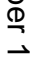


TABLE I

Situation of Growth in i, 500 Consecutive Combined Excision Specimens of Carcinoma of the Rectum and Rectosigmoid Examined at St. Mark's Hospital, 1933-47.

\begin{tabular}{|c|c|c|c|c|}
\hline \multicolumn{2}{|c|}{$\begin{array}{l}\text { Distance of lower edge } \\
\text { of growth from anus on } \\
\text { sigmoidoscopy }\end{array}$} & $\begin{array}{c}\text { Number of growths } \\
\text { at each level }\end{array}$ & $\begin{array}{l}\text { Percentage of growths } \\
\text { at each level }\end{array}$ & $\begin{array}{l}\text { Percentage of intra and } \\
\text { extra peritoneal growths }\end{array}$ \\
\hline \multicolumn{2}{|c|}{$\begin{array}{l}\text { Inches } \\
\text { I6 }\end{array}$} & 2 & $\ldots$ & - \\
\hline & & .. & - & 二 \\
\hline \multicolumn{2}{|c|}{14} & 2 & - & - \\
\hline \multicolumn{2}{|c|}{13} & 5 & - & - \\
\hline & & 7 & - & - \\
\hline \multicolumn{2}{|c|}{11} & 27 & 1.80 & \\
\hline & & 33 & 2.22 & \\
\hline \multirow{2}{*}{\multicolumn{2}{|c|}{$\begin{array}{l}9 \\
8\end{array}$}} & $\begin{array}{l}70 \\
8 I\end{array}$ & 4.67 & 47.53 per cent. \\
\hline & & & $\begin{array}{l}5.4^{\circ} \\
8.00\end{array}$ & \\
\hline \multicolumn{2}{|c|}{$\begin{array}{l}7 \\
6\end{array}$} & 160 & $\begin{array}{r}0.00 \\
10.67\end{array}$ & \\
\hline \multicolumn{2}{|c|}{5} & 206 & 13.67 & \\
\hline \multirow{4}{*}{$\begin{array}{l}\text { Average site } \\
\text { peritoneal } \\
\text { reflection }\end{array}$} & $\begin{array}{c}\text { Inches } \\
4\end{array}$ & 205 & 13.67 & ? \\
\hline & 3 & 198 & 13.20 & 52.47 per cent. \\
\hline & 2 & 188 & 12.53 & extra-peritoneal \\
\hline & I & 196 & 13.07 & $x^{\circ}+2+2$ \\
\hline
\end{tabular}

N.B.-Up to 1947 it was customary at St. Mark's Hospital to perform a Paul-Mikulicz resection and not a combined excision for growths situated more than II in. from the anus hence the small numbers found at higher levels in this series of combined excision specimens.

mesocolon on the marginal artery close to the sigmoid colon. To cope with all these possible extensions obviously nothing short of the wide removal of rectum and related structures provided for in the now classical abdomino-perineal excision devised by him would suffice, and his work therefore left no place for sphincter preservation in the radical treatment of rectal cancer.

Subsequent workers, however, and notably Cuthbert Dukes (1930, 1940) and Heinrich Westhues (1930, 1934) who have reinvestigated the mode of spread of rectal cancer as revealed by dissection of many hundreds of operative specimens removed by perineal or combined excision, paint a rather different picture. They agree with Miles in showing that the main avenue of lymphatic extension is upwards along the superior haemorrhoidal and inferior mesenteric vessels, but find that the paracolic glands in the sigmoid mesocolon are seldom implicated. As for downward spread in the extramural lymphatics below the level of the primary growth, this in their experience is very rare except when the glands of the upgoing lymphatics are choked with carcinoma cells and the case is virtually incurable anyway. Lateral lymphatic spread along the levator muscles could not be verified as a rule because, contrary to the illustrations of Miles (1926), excision specimens seldom contain any glands in this region. Finally spread in rectal wall itself either by direct extension or in the submucous lymphatic plexus is, except in highly anaplastic growths, a very limited proces seldom reaching more than 1.5 to $2 \mathrm{~cm}$. beyon the palpable edge of the primary carcinomatous ulcer.

Their work then would suggest that in excising growths of the upper half of the rectum-and ever more so those of the rectosigmoid-it should not be necessary to remove more than $I \frac{1}{2}$ in. or so of rectal wall and perirectal fat and lymphatics distaf. to the lower edge of the growth, nor to sacrifices the sigmoid colon and mesocolon extensively asn recommended by Miles. It may be considered tơ justify, nay demand, a retrial of sphincter-saving operations for these higher rectal and rectosigmoids growths. On the strength of these investigation of many surgeons, including Pannett (1935), Babcock (1932, 1940), Bacon (1945), Dixon (1939Nㅡㄴ 1944) and Wangensteen (1943, 1945), have turneh their attention to this type of operation, and क group of us at St. Mark's Hospital have also con- $\omega$ ceived it as our duty to test these operations in actual practice especially since the advent of sulph drugs and antibiotics to minimize the risks of sepsis. W have now treated some 146 cases by these procedures and this paper is an account of our experiences in their management. 


\section{Selection of Cases for Sphincter-Saving Resections}

The site of the growth has been the main criterion in deciding on the suitability of a case for a sphincter-preserving excision. As already mentioned, we consider that extraperitoneal growths of the rectum call for a combined excision and should not be treated by resection. We have, therefore, reserved resection for growths situated at or above the level of the peritoneal reflection off the front of the rectum. Actually this reflection is a rather variable landmark, but usually it lies about 8-ro cm. on sigmoidoscopy or roughly as far as the finger can feel on digital examination of the rectum. We have, therefore, taken it as a rule that growths lying at a lower level than this, which can easily be felt with the examining finger, are too low for resection.

Not every growth above this level encountered by the three or four surgeons contributing to this series of cases was in fact treated by resection. All the rectosigmoid growths and most of the intraperitoneal rectal growths were, but the nearer the lesion lay to the peritoneal reflection the greater were the technical difficulties and sometimes these made it impossible to carry out a resection. Thus in a few cases a combination of a narrow pelvis and a bulky fat-laden sigmoid colon and mesocolon, rendering intrapelvic suturing difficult, decided us against a sphincter-preserving operation. In some others the presence of a double growth or marked diverticulosis necessitating sacrifice of an unusually large amount of colon has put resection with anastomosis out of court, and a combined excision has had to be performed instead. With increasing experience of the technique, however, we have less frequently been baulked by difficulties of this sort.

It should be emphasized that, at any rate for the greater part of this series, no attempt has been made to follow the recommendation of Grey Turner (1935) and select for resection only those cases adjudged clinically and at operation to have specially early growths. We have rejected this policy for two reasons: firstly, because we are convinced that it is impossible to decide, prior to pathological examination of the excised specimen, how extensively any growth has spread, bitter experience having shown us on many occasions that carcinomata which we thought were in a very early stage of development were in fact associated with extensive lymphatic metastases, and, secondly, because it implies that resection operations are procedures of less radical scope than a formal combined excision. As we understand it, on theoretical grounds a resection should secure as thorough an eradication of growths of the upper rectum and rectosigmoid as an abdomino-perineal excision, but if in practice it is demonstrated that it does not do so, then it should be discarded entirely as a radical procedure.

Finally, special mention should be made of the cases with hepatic metastases. It has been our practice when operating on carcinomata of the rectum and other parts of the gastro-intestinal tract to carry out palliative excision of the primary growth even when liver second iries are present, provided that not more than a half of the liver substance has been replaced by tumour tissue, because these patients often survive for twelve months or two years subsequently and their symptomatic condition in that remaining period of life is enormously improved if the primary lesion has been removed. Obviously the palliative value of excision in cases of this kind with rectal growths is greatly enhanced if it can be carried out as a resection without sacrifice of the sphincters. When we find, therefore, that hepatic deposits are present in a case of carcinoma of the rectum we make every effort to perform a resection rather than an ordinary excision, stretching the indications to include rather lower growths than we would normally be prepared to accept for resection with a view to cure, and in consequence shouldering much greater technical difficulties.

\section{Choice of Operation}

Sacral Resection. A very large number of resec? tions of this type have been performed in Germany and Austria where owing to the advocacy of Kraske (1885), Hochenegg (1888, 1889), Küttner (1910, 1916) and Mandl (1932, 1945), this was for many years the most popular form of resection for rectal carcinoma in some of the leading surgical clinics, and Hochenegg and Küttner alone were responsible for nearly a thousand of these operations. We have not employed this method because it has seemed to us an insufficiently radical procedure in that, through a purely sacral wound, it is impossible to carry out a really high ligation of the superior haemorrhoidal-inferior mesenteric vascular pedicle. It was for this reason that the perineal excision of LockhartMummery was quite properly abandoned at St. Mark's Hospital in favour of the combined abdomino-perineal or perineo-abdominal operation. In turning to resection operations, therefore, it was logical that we should try to develop methods incorporating an abdominal phase to permit of adequate clearance of the upwardtraining lymphatic channels.

Abdomino-sacral Resection. This operation has been extensively practised by Finsterer (194I), Goetze (1944), and d'Allaines (1950) and was the first method recommended by Pannett (r935). We have used it on only three occasions and 
found that in all the patients the sacral wound broke down to some extent, in two of them resulting in the formation of a fistula between the bowel and the skin, despite the fact that a preliminary transverse colostomy had invariably been established to defunction the rectum. Finsterer (194I) and d'Allaines (1950) do not seem to have had this trouble, but Goetze (I944) recognizes it as one of the main problems of this operation, and it is significant that Pannett (195I) has now given up this technique and gone over to a purely abdominal resection.

Abdomino-anal Resection. The most popular operation of this type is the 'pull through' operation of Babcock (1947) and Bacon (1945). In this procedure as usually practised the anal mucosa is excised and the sphincter muscles divided and resutured round the sigmoid colon, which has been drawn through the anus after resection of the rectum. These manoeuvres, however, have the effect of damaging or completely destroying the sensory and motor components of the delicate sphincter apparatus and as a consequence continence is grossly impaired. An examination of four patients operated on by other surgeons with this technique left us in no doubt that after this operation patients are not usually properly continent but have really anal colostomies, and we did not feel that these functional results justified the use of this particular technique.

An alternative method of performing abdominoanal excision which we have used in 26 cases is that associated with the names of Maunsell (1892) and Weir (rgor). Here after an abdominal dissection the rectum is resected leaving a long colon stump and short ano-rectal stump. The latter is then turned inside out through the anus so that its cut upper margin becomes the projecting lower edge, and the colon stump is drawn down through it so that the free edges of both stumps lie opposite one another and can be sutured together outside the anus. Finally the anastomosis is returned through the anus. The surgeon is thus enabled to perform a very low resection and anastomosis without a perineal or sacral wound, and without dividing the sphincters or damaging the mucosa of the anal canal or lower rectum. The functional results are accordingly very much better than after the Bacon-Babcock operation, and, though a few of the patients have had some difficulty in controlling flatus and liquid faeces at first, the majority are fully continent. Our main criticism of this operation is that it requires a particularly long piece of colon to stretch to the anal region and we have not-always been able to manufacture a well vascularized colon stump of sufficient length. Also the colo-rectal anastomoses in these cases seem specially liable to break down in part, probably due to post- 3 operative thrombosis in the vessels supplying the $\stackrel{\varnothing}{\propto}$ long piece of colon.

Abdominal or Anterior Resection. This is the $\overrightarrow{\vec{B}}$ technique which we have chiefly employed, and following the important papers of Wangensteen $(1943,1945)$ we have used it almost to the complete $\frac{\bar{c}}{\mathrm{~N}}$ exclusion of other methods of resection. Accept- $\vec{\nabla}$ ing as we do only intra-peritoneal rectal and recto- $\propto$ sigmoid growths for resection, we find that we can is deal with these growths perfectly satisfactorily by $\vec{\circ}$ anterior resection without having to resort to an abdomino-sacral or -anal technique to complete $\vec{\omega}$ the anastomosis. It is undoubtedly an advantage $\frac{O}{0}$ to be able to avoid these latter methods because after the purely abdominal resection healing of the anastomosis is much more certain, and, due to the larger rectal remnant left, the functional result is always perfect. It is only very occasionally now when doing a palliative resection for a rather lower growth than usual that we find it necessary to use the abdomino-anal technique. Altogether we have treated 120 cases by anterior resection employing the following technique with slight modifications according to the individual surgeon.

\section{Technique of Anterior Resection}

Pre-operative Care. The preparation of patiento for anterior resection does not differ essentiall $\bar{\phi}$. from that employed for an ordinary combined excision. Loçally it is designed to clear the bowel of faeces retained above the growth, and to sterilize the gut contents by intestinal antiseptics. The former aim is secured as far as possible by administration of aperients such as Milpar to soften the faeces and assist in their expulsion through the carcinomatous stricture, and by twice daily enemata or washes-out. It must be admitted, however, that if the growth is completely annular and tightly constricting, this mechanical cleansing is often very ineffective even if prolonged over a period of a fortnight or three weeks instead of the usual seven to ten days set aside for preparation of these cases. The intestinal antiseptic used in most of our cases was sulphasuccidine (20 gm. daily in divided doses every four hours) or sulphathaladine (ro gm. daily in divided doses) for five to seven days, but more recently this has been supplemented by streptomycin, I gm. orally b.i.d. for the last two or three days. Terramycin or aureomycin are perhaps even more effective than streptomycin but they have not been readily available to us. We have recently started using chloromycetin and it may prove to be the best drug of all for this purpose.

In addition to this local preparation the patients' general condition is carefully assessed, and anaemia 
and hypoproteinaemia are rectified by preoperative blood transfusion and by high protein diet supplemented by protein concentrates such as Casilan. All patients are given vitamin B and $\mathrm{C}$ preparations as a routine to correct any vitamin deficiency.

Operative Technique. We like to have the patient in the lithotomy-Trendelenburg position for this operation because, if the growth should be shown at laparotomy to be unsuitable for an anterior resection, it is an easy matter with the patient so positioned to change the operative procedure to an abdomino-anal resection or a synchronous combined excision as required. Also in this position it is possible for the rectum to be washed out per anum during the course of the operation just before it is divided below the growth, which greatly reduces the risks of soiling and dissemination of loose carcinoma cells in the pelvis.

The operation is perfarmed through a long left paramedian incision extending from the pubis to $I_{2} \frac{1}{2}$ in. above the umbilicus. The steps of the dissection follow those of the abdominal phase of the abdomino-perineal excision the rectum being mobilized posteriorly, anteriorly and then laterally as far almost as the anorectal ring. If, however, the growth is situated high in the rectosigmoid it is not necessary to separate the extraperitoneal part of the rectum from the sacral concavity or to divide the lateral ligaments. The inferior mesenteric vessels are then tied and divided; it is usually possible to apply this ligature just below or above the origin of the first sigmoid artery. The sigmoid mesocolon is then divided obliquely from this point to the colon which is severed between crushing clamps, leaving proximally a stump of sigmoid colon and mesocolon nourished by the first sigmoid or the descending branch of the left colic artery (Goligher, 1949). Attention is now directed to dividing the rectum $1 \frac{1}{2}$ in. to 2 in. below the growth. As a preliminary the mesorectum is separated from the back of the rectum at this level by blunt dissection and divided between clamps. The bowel is thus bared all round for a segment of an inch or so. A curved Parker-Kerr crushing clamp is next applied to the upper limit of the bared zone, and about $I_{2} \frac{1}{2}$ in. below the growth, and an assistant then passes a proctoscope per anum, irrigates the closed-off lower compartment of the rectum with $\mathrm{I} / 500$ perchloride of mercury for five minutes and finally swabs it dry. The operation is then continued by applying another Parker-Kerr clamp just below the first one and dividing between them; this completes the resection of the carcinomatous segment of bowel.

The clamps holding the colon and rectal stumps are now approximated and a posterior row of
Lembert sutures of fine interrupted Deknate inserted. The clamps are then removed, the crushed edges of rectum and colon trimmed off? and a continuous through and through stitch of fine No. 2/0 chromic catgut, mounted on a sma焦 half circle atraumatic needle on a needle holder inserted between the colon and rectum, the pose terior half being introduced as a simple over an over stitch, the anterior as a Connell stitch t $\bar{\phi}$ secure good inversion of the mucosa. Th\& anastomosis is completed by an anterior row of Lembert sutures of fine Deknatel. Finally the pelvic peritoneum is stitched to the colon 2 or 3 in. above the bowel suture line, which has the effecty of placing the entire anastomosis below the perie toneum. The extraperitoneal pelvic space is: drained extraperitoneally by a strip of corrugated rubber taken out below the reflection of pelvic peritoneum at the side of the bladder and through the lower end of the main paramedian wounder Penicillin sulphonamide powder is dusted locall $\$ 0$ around the anastomosis. The abdomen is close $\phi$ in layers usually with nylon or stainless steel wire.

In our earlier cases a preliminary transvers colostomy was created two or three weeks befor the main resection and irrigations and sulphaz succidine suppositories given into the distal lim daily to cleanse and sterilize the left half of the colon and rectum. Then for a time we made

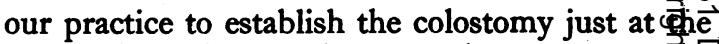
end of the main resection operation to protect the anastomosis from undue strain, but more recentle and for quite the majority of our cases, a colose tomy has been avoided entirely with apparentlo no greater incidence of serious complications. A the present time we reserve transverse colostom $\vec{\delta}$ for the few cases where the anastomosis has proved exceptionally difficult or the colon has been unduly loaded. Also in three cases, where a simultaneous Wertheim's hysterectomy was performed with anterior resection of the rectum? a transverse colostomy was established to lesse the risk of a rectovaginal fistula developing if the bowel suture line should break down: In the cases not having colostomy we used to pass a rectal tube from the anus into the sigmoid colon? the point of the tube being guided through the anastomosis by the operator before the abdoment. was shut. This was retained for four or five daysu Alternatively the anal sphincters were vigorously stretched to paralyse them and prevent the accue mulation of flatus in the rectum under tension But in most cases now these steps are all omitted

With suitably long retractors, scissors, needlee holders and other instruments and experience of the technique this is not a difficult operation, but it does usually take a little longer to complet than the average synchronous combined excision? 
Our anaesthetists and registrars, however, are unanimous in their opinion that the condition of the patients at the end of these operations is distinctly better than after a formal combined excision, presumably because of the avoidance of the haemorrhage and shock associated with the perineal phase of the latter procedure.

Post-operative Care and Complications. Blood is administered during and after operation as required, and an intravenous infusion of saline or dextrose water is usually continued for two or three days, till flatus is passed or peristaltic sounds return to normal. As mouth feeds are gradually built up and light diet is resumed the bowels usually act spontaneously about the fourth or fifth day. A routine in all these cases is, of course, chemotherapy with oral or intramuscular sulphamezathine I gm. four-hourly, penicillin 500,000 units b.d., and intramuscular streptomycin $0.5 \mathrm{gm}$. b.i.d. In addition, when the colon is sectioned at operation a sample of the faeces is cultured for any organism surviving the pre-operative intestinal antisepsis and the sensitivity of such organisms to various antibiotics is determined as a guide to further treatment if required.

The suprapubic drain is left in situ for at least five days. There is often a good deal of discharge of blood-stained fluid along it for the first day or so, later this dries up or may become slightly purulent or, in about one case in twelve, frankly faecal, indicating that the suture line in the bowel has broken down. However, even if a faecal fistula should occur this nearly always closes spontaneously in 10 to I4 days and only rarely has it been necessary to establish a temporary transverse colostomy to get it to close.

If the suture line in the bowel is gently palpated or inspected sigmoidoscopically at the end of a week, it is found in about one case in six to have broken down at some point on its circumference, usually the posterior third, and the rectal lumen then communicates with an extrarectal presacral cavity. Only in about a half of these cases does faeces escape suprapubically, and in any event the defect rapidly closes so that at the end of two-and-a-half or three weeks it is represented only by a slight dimple on the suture line.

If a transverse colostomy has been made an enterotome is applied to the colostomy spur at the end of 10 to 14 days, provided that the temperature has returned to normal and there is no evidence of gross pelvic sepsis. When the spur has been completely obliterated the colostomy opening is finally closed extraperitoneally.

One of the striking and unexpected features about the convalescence from these operations has been the relative freedom from serious sepsis, which is presumably due in part to the use of antibiotics. In four cases, however, pelvic ab- $\frac{3}{\varnothing}$ scesses did develop; these discharged into the $\stackrel{\odot}{\circ}$ lumen of the rectum through the bowel suture $\stackrel{.}{~}$ line or suprapubically, and one burst into the $\overrightarrow{\bar{F}}$ vagina as well giving the patient a temporary recto-vaginal fistula which fortunately closed $\frac{5}{0}$ spontaneously.

Another impressive thing about these cases has $\underset{\mathbb{Q}}{\mathbb{Q}}$ been the fact that, though there is often considerable narrowing at the suture line at the conclusion of the operation, this never results in a permanent. organic stenosis. Actually, if the patients are $\overrightarrow{\vec{\omega}}$ re-examined at the end of three months, though $\stackrel{\sigma}{\omega}$ the lumen has opened up considerably, the suture $\vec{C}$ line is still evident as a slight fibrous constriction, 3 but on further examination after another six or $i$ twelve months no narrowing usually remains and $\dot{\omega}$ it may indeed be difficult to identify the colo- $\vec{\omega}$ rectal junction with confidence. Therefore, no $\delta$ special operative technique or post-operative $\infty$ dilatation is required to guard against stenosis; 은 the patients' own faeces are a sufficient dilator.

As already mentioned, after anterior resection $z$ even when this is carried as low as is technically feasible, the patients invariably enjoy perfect control of flatus and faeces, but owing to the loss of part of the sigmoid colon in which faecs $\overrightarrow{0}$ are normally accommodated, more frequer of motions are required, usually two or three a d at least.

\section{Operative Mortality}

Eleven of the 120 cases subjected to anterior $\frac{2}{8}$ resection died and also one of the 26 patients treated by abdomino-anal excision, giving an $\frac{0}{3}$ overall majority of 12 or 8.2 per cent. in a total of $\frac{\partial}{\partial}$ 146 sphincter-saving resections. This compares satisfactorily with the current immediate mortality rate for combined excision in St. Mark's 3 Hospital of approximately 7 per cent. The causes of death are shown in Table 2. It should be especially noted that only two cases died of sepsis. $\stackrel{8}{\partial}$

\section{TABLE 2}

Causes of Operation Deaths after ANTERIOR OR믐 Abdomino-Anal Resection

$\begin{array}{llllllll}\text { Shock } & \ldots & \ldots & \ldots & \ldots & \ldots & \ldots & 2 \text { cases } \mathcal{N}\end{array}$

$\begin{array}{llllll}\text { Coronary occlusion } & \ldots & \ldots & \ldots & \ldots & 2 \text { cases } \\ \end{array}$

Paralytic ileus and cardiac failure $\quad \ldots \quad \ldots$ I ,

Perforated gastric or duodenal ulcer ... . . 2 cases $N$

Pulmonary embolus $\quad \ldots \quad \ldots \quad \ldots \quad \ldots \quad$ I case

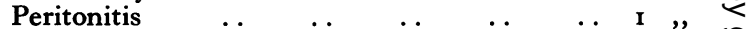

Intestinal obstruction and bronchitis .. $\quad . . \quad$ I,$"$ 을

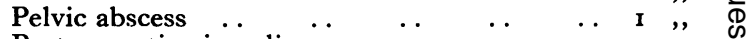

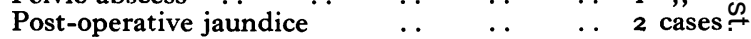

I 2 cases 
ORIGINAL RESECTION SPECIMEN

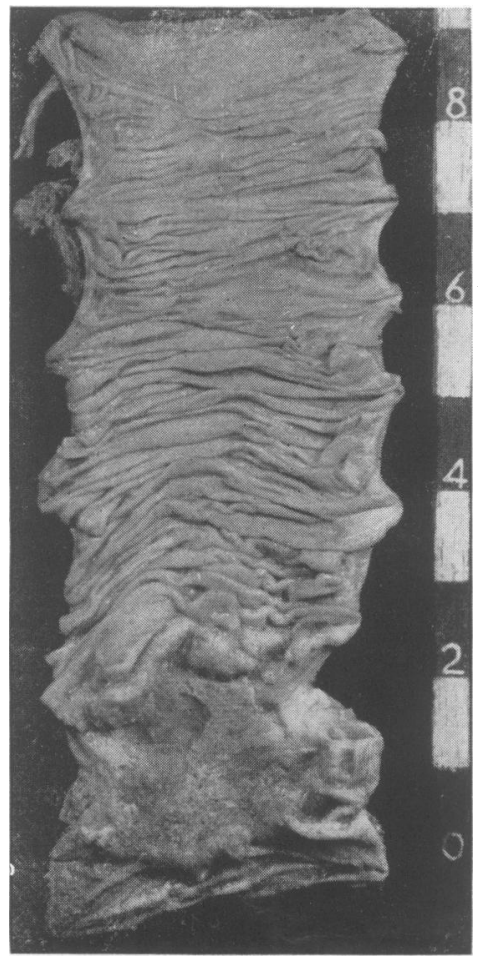

FIG. I.
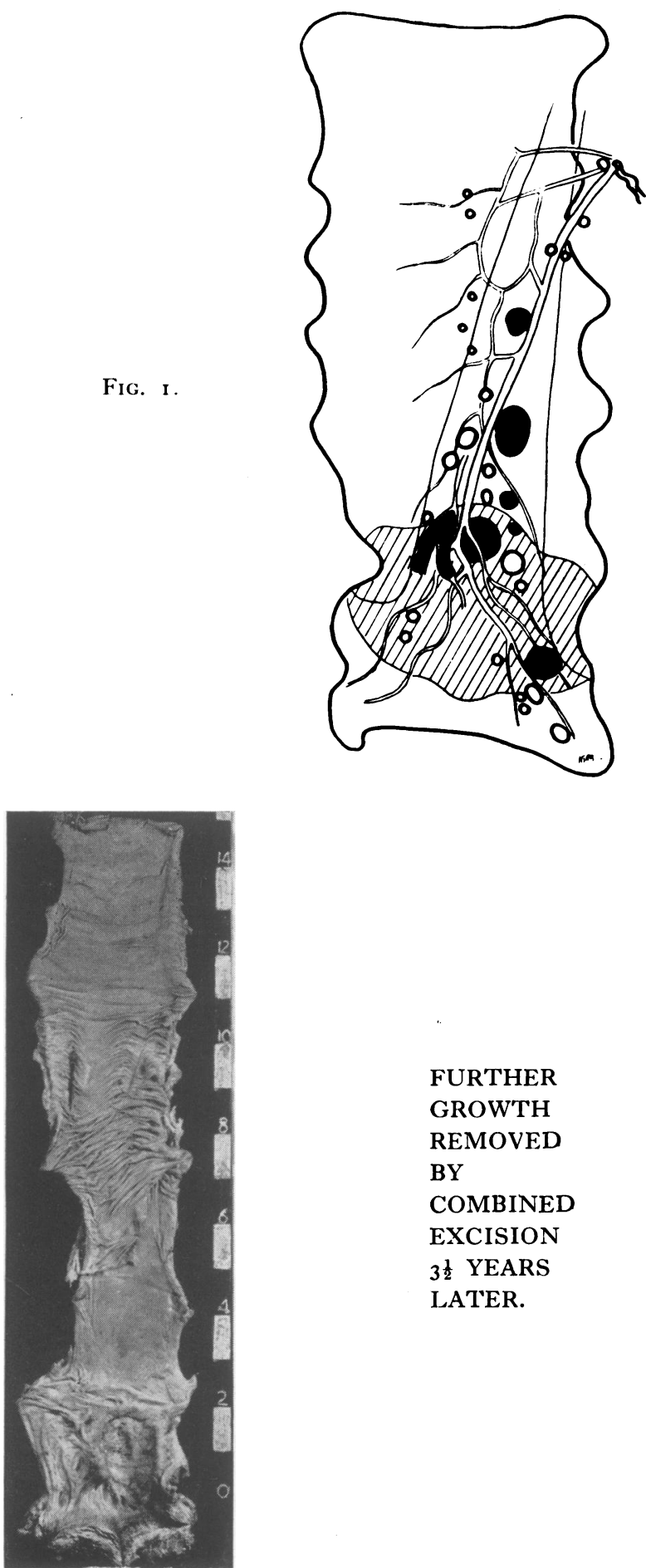

FURTHER

GROWTH

REMOVED

BY

COMBINED

EXCISION

$3 \frac{1}{2}$ YEARS

LATER.

FIG. 2.-Recurrence (?) fresh primary growth developing three years and nine months after anterior resection for carcinoma recti. The second growth arose in the ampulla of the rectal stump posteriorly just below the colo-rectal suture line. 


\section{Late Results}

While the immediate results of these resection operations have been most encouraging, it must be admitted frankly that the remote results have caused disappointment. Of the 134 cases treated by anterior resection, already in have developed local recurrences, and as most of these operations were performed in the last three or four years further recurrences may reasonably be expected in the more recently treated cases. A detailed report on these recurrences and others is being made by the writer in conjunction with Cuthbert Dukes and H. J. R. Bussey (I95I). It suffices to say here that usually the recurrence appeared in the bowel wall, most often at the site of the anastomosis, sometimes in the rectal stump as in Fig. 2. They have appeared at any time from three or four months to six or seven years after. It is quite possible that some of them may be fresh primary growths, whilst others may be due to implantation on the suture line of loose carcinoma cells mixed with the faeces in the lumen of the rectum, hence the precautions which we now take to cleanse the rectum with perchloride of mercury before dividing it. But presumably many of them are ordinary recurrences due to inadequate removal. Before condemning the operation, however, it is important to realize that local recurrences are also sometimes found after ordinary combined excision, and, owing to the greater difficulty of demonstrating them clinically in these cases are probably present more frequently than is generally imagined.
We do, of course, know the results of the combined excision in terms of five-year survival rate but similar figures cannot yet be worked out $\stackrel{\varrho}{ }$ for anterior resection, and three or four years will $\stackrel{4}{-}$ have to elapse before this comparision can be $\overrightarrow{\bar{A}}$ made. It will be seen, therefore, that sufficiento data are not yet available to enable one to deter-드 mine the precise value of these resection operations $\overline{\bar{\sigma}}$ in the treatment of carcinoma of the upper rectum $\Phi$ and rectosigmoid. The most that one can say at the moment is that they are excellent palliative ${ }^{\text {s }}$ procedures for patients with growths in either $\vec{\circ}$ of these situations who are having an excision $\overrightarrow{\vec{\omega}}$ done in the presence of hepatic metastases $\stackrel{\omega}{\sigma}$ (and who represent approximately 10 per: cent. of the cases having rectal excision done at 3 . St. Mark's Hospital). It is highly likely that resection will retain its place in the management ${ }_{\omega}$ of these incurable cases because even if local $\vec{\omega}$ recurrences should develop subsequently the patients will probably succumb from their liver $\infty$ secondaries before the local recurrence causes 음 serious symptoms. But for other cases the claims of this operation to be considered as a radical $Z$ procedure on a par with the orthodox combined excision remain to be substantiated.

The writer is greatly indebted to his colleague at St. Mark's Hospital for kindly permitting hif $\overrightarrow{0}$ to include their cases in this survey. He also wishes to record his thanks to the British Empin Cancer Campaign a grant from which defrayed some of the expenses involved in this work.

\section{BIBLIOGRAPHY}

ALLEN, A. W. (1946), Surg. Gyner. E๐ Obstet., 82, 490. BABCOCK, W. W. (1932), Ibid., 55, 627.

BABCOCK, W. W. (r940), in "The Treatment of Cancer and Allied Diseases,' by G. T. Pack and E. M. Livingstene, New York, P. Hoeber, ${ }_{5} I_{3}$.

BABCOCK, W. W. (1947), Surg. Gynec. E Obstet., 85, I. BACON, H. (1945), Ibid., 81, I 13.

D'Allaines (1950), Proc. Roy. Soc. Med., 43, 697.

DIXON, C. F. (1939), Amer. F. Surg., 46, 12.

DIXON, C. F. (1944), Surgery, 15, 367.

DUKES, C. E. (1930), Brit. F. Surg., 17, 643 .

DUKES, C. E. (1940), F. Path., 50, 527.

FINSTERER, H. (r94I), Arch.f. klin Chir., 202, 15.

GABRIEL, W. B. (1948), 'The Principles and Practice of Rectad Surgery,' 4th edition, H. \& K. Lewis, London.

GOETZE, O. (1944), Arch. f. klin. Chir., 206, 293.

GOLIGHER, J. C. (1949), Brit. F. Surg., 37, 157.

GOLIGHER, J. C., DUKES, C. E., and BUSSEY, H. J. R. (1951). Brit. F. Surg. (in the press).

HOCHENEGG, J. (1888), Wien klin. Wschr., 1, 254, 272, 290, 300. 324,348 .

HOCHENEGG, J. (1889), Ibrid., 1, 578.
KRASKE, P. (1885), Verhandl $d$ deutsch. Gesellsch $f$ Chir., Berl. I4, P. 2, 464

KUTTNER, H. (1910), Deutsch Med. Wschr., 36, 606.

KUTTNER, H. (r916), Zbl. Chir., 43, 905.

MANDL, F. (1932), Ibid., 394.

MANDL, F. (1945), Surgery, 18, 3 I 8.

MAUNSELL, H. W. (1892), Lancet, 2, 473.

MILES, W. E. (1910), Brit. Med. Fournal, 2, 940.

MILES, W. E. (1926), 'Cancer of the Rectum,' Harrison \& Sons, London.

PANNETT, C. A. (1935), Lancet, 2, 423

PANNETT, C. A. (I95I), personal communication.

RANKIN, F. W. (1947), Ann. Surg., 126.

TURNER, G. GREY (1935), Proc. Roy. Soc. Med., 28, 55.

WANGANSTEEN, O. H. (1943), Surgery, 14, 403.

WANGANSTEEN, O. H. (1945), Surg. Gyneç. छ Obstet., 81, 1.

WEIR, R. F. (1901), F.A.M.A., 37, 801.

WESTHUES, H. (1930), Arch. f. klin. Chir., 161, 582.

ESTHUES, H. (1934), ' Die Pathologish-Anatomischen Grund- N lagen der Chirurgie des Rectumkarzinoms,' Georg 'Thieme Verlag, Le:- zip. 